\title{
Beatmungsassoziierte Pneumonie - Therapie
}

\section{Ventilator-associated pneumonia - treatment}

In Deutschland gibt es jährlich circa 200000 Erkrankungsfälle an nosokomialer Pneumonie. Die Sterblichkeit kann - vor allem bei Patienten, die auf einer Intensivstation behandelt werden - bis zu $50 \%$ betragen. Dabei kann die direkt auf die Pneumonie zurück zu führende Letalität ebenfalls bei bis zu $50 \%$ liegen $(1,2)$. Eine allgemein akzeptierte Standardtherapie der beatmungsassoziierten Pneumonie gibt es nicht. Die initiale antimikrobielle Therapie muss in Unkenntnis des zugrundeliegenden Erregers immer als kalkulierte Therapie begonnen werden. Für die initiale antibiotische Therapie ist von großer Bedeutung, ob eine nosokomiale Pneumonie früh, also innerhalb der ersten 4 Tage nach der stationären Aufnahme (Erregerspektrum: Staphylococcus aureus, Haemophilus influenzae, Streptococcus pneumoniae, Enterobacteriaceae) oder später (Erregerspektrum: zusätzlich MRSA, Pseudomonas spp., Acinetobacter spp., Enterobacter spp., Proteus vulgaris und Serratia spp.) entsteht. MRSA (multiresistenter Staphylococcus aureus) spielt in der Regel nur bei spät auftretenden Pneumonien eine Rolle. Bei einer örtlichen Häufigkeit von mehr als 15\% MRSA sollte primär die Gabe eines Glykopeptids, eines Streptogramins oder eines Oxazolidinons erwogen werden. Tab.1 zeigt Erreger bakterieller beatmungsassoziierter Pneumonien, die in mehreren Studien evaluiert werden konnten.

Besondere Bedeutung und allgemeine Akzeptanz für die Therapie nosokomialer Pneumonien hat die Klassifizierung der American Thoracic Society (ATS) aus dem Jahre 1995 erhalten (Abb.1). Hier werden primär leichte bis mittelschwere von schweren Pneumonien unterschieden (3).

Die beatmungsassoziierte Pneumonie gehört definitionsgemäß zu den schweren Verlaufsformen, daher wird im Folgenden nur auf die Klassifikationen der schweren Pneumonien aufgeführt (Gruppe 1 und Gruppe 3). Bei schwerer Erkrankung ohne Risi-
Tab. 1 Keime, die bei Patienten mit beatmungsassoziierten Pneumonien isoliert wurden

\begin{tabular}{|c|c|c|c|}
\hline Pathogen & $\begin{array}{l}\text { Rello et al. } \\
n=129\end{array}$ & $\begin{array}{l}\text { Torres et al. } \\
n=78\end{array}$ & $\begin{array}{l}\text { Fagon et al. } \\
n=52\end{array}$ \\
\hline \multicolumn{4}{|l|}{$\begin{array}{l}\text { Aerobe Gram } \\
\text { positive Keime: }\end{array}$} \\
\hline Staphylococcus aureus & $25 \%$ & $9 \%$ & $20 \%$ \\
\hline $\begin{array}{l}\text { Streptococcus } \\
\text { pneumoniae }\end{array}$ & $4 \%$ & nicht genannt & $3 \%$ \\
\hline Enterococcus spp. & $2 \%$ & $4 \%$ & nicht genannt \\
\hline andere & $2 \%$ & $4 \%$ & $14 \%$ \\
\hline \multicolumn{4}{|l|}{$\begin{array}{l}\text { Aerobe Gram } \\
\text { negative Keime: }\end{array}$} \\
\hline Haemophilus influenzae & $18 \%$ & nicht genannt & $6 \%$ \\
\hline $\begin{array}{l}\text { Pseudomonas } \\
\text { aeruginosa }\end{array}$ & $21 \%$ & $22 \%$ & $19 \%$ \\
\hline Acinetobacter spp. & $3 \%$ & $39 \%$ & $9 \%$ \\
\hline Proteus spp. & $3 \%$ & $4 \%$ & $9 \%$ \\
\hline Serratia spp. & $4 \%$ & $4 \%$ & $9 \%$ \\
\hline Klebsiella spp. & nicht genannt & nicht genannt & $2 \%$ \\
\hline Escherichia coli & $3 \%$ & nicht genannt & $5 \%$ \\
\hline andere & $7 \%$ & $8 \%$ & $9 \%$ \\
\hline Anaerobe Keime & $3 \%$ & nicht genannt & $1 \%$ \\
\hline Fungi & $3 \%$ & $4 \%$ & nicht genannt \\
\hline
\end{tabular}

kofaktoren und frühem Manifestationszeitpunkt („early onset“, innerhalb der ersten 4 Tage nach Aufnahme) erfolgt die Einteilung in die Therapiegruppe 1. Patienten ohne Risikofaktoren mit spätem Erkrankungsbeginn („late onset“) werden der Gruppe 3 zugeordnet. Patienten mit Risikofaktoren fallen unabhängig 


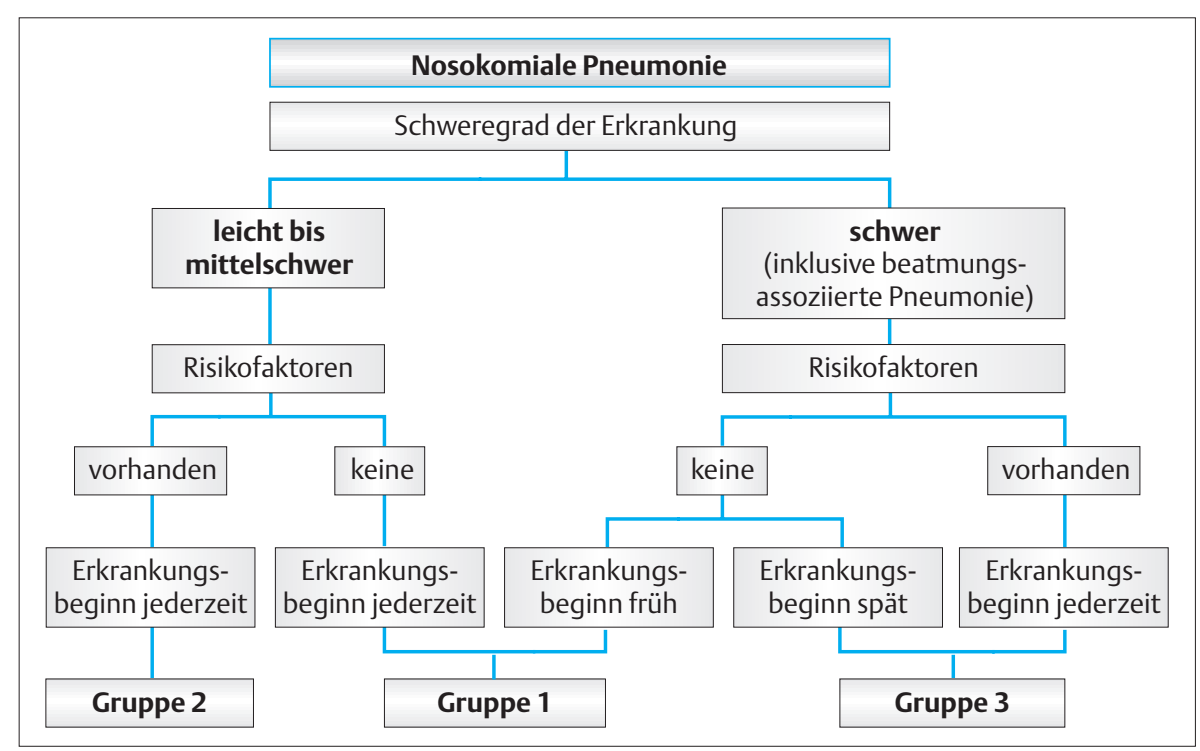

Abb.1 Klassifizierung der nosokomialen Pneumonien durch die American Thoracic Society 1995 (3). Patienten mit beatmungsassoziierter Pneumonie werden definitionsgemäß den schweren Verlaufsformen (Gruppe 1 oder Gruppe 3) zugeordnet.

Nosokomiale Pneumonie (Gruppe 1)*

- mittelschwere Erkrankung, keine Risikofaktoren, zu jeder Zeit - schwere Erkrankung (inklusive beatmungsassoziierte Pneumonie), keine Risikofaktoren, „early onset“

\begin{tabular}{l}
\multicolumn{1}{|c|}{ häufigste Erreger } \\
S. pneumoniae \\
S. aureus (MSSA) \\
Hämophilus influenzae \\
E. coli \\
Klebsiella spp. \\
Proteus spp. \\
Serratia spp.
\end{tabular}

\begin{tabular}{l}
\hline \multicolumn{1}{|c|}{ Antibiotikatherapie } \\
Cephalosporin II, \\
evt. Cephalosporin III \\
ohne P. aeruginosa-Aktivität \\
Acylureidopenicillin \pm Inhibitor oder \\
Aminopenicillin \pm Inhibitor, \\
ohne P. aeruginosa-Aktivität
\end{tabular}

Abb.2 Erreger nosokomialer Pneumonien und Therapie bei Patienten, die der Gruppe 1 zugeordnet werden (in Anlehnung an das Consensus Statement der American Thoracic Society (3)). Patienten mit beatmungsassoziierter Pneumonie werden definitionsgemäß den schweren Verlaufsformen zugeordnet.

vom zeitlichen Beginn in die Gruppe 3. Für die nosokomialen Pneumonien, die entsprechend der Therapieempfehlungen für Gruppe 1 behandelt werden sollen (mittelschwere Erkrankungen, ohne Risikofaktoren, Beginn zu jeder Zeit sowie schwere Erkrankungen ohne Risikofaktoren, „early onset“), werden Cephalosporine der 2. Generation ohne Pseudomonas-Wirksamkeit oder Amino- bzw. Acylureidopenicilline in Kombination mit einem Betalaktamase-Inhibitor vorgeschlagen (Abb.2).

Die der Gruppe 3 zugeordneten Patienten bekommen nach den Richtlinien der American Thoracic Society eine Kombinationsbehandlung bestehend aus einem pseudomonaswirksamen Betalaktam-Antibiotikum in Kombination mit einem Fluorchinolon oder einem Aminoglykosid. Als Betalaktam-Antibiotika mit Pseudomonasaktivität stehen Piperacillin/Tazobactam, Ceftazidim, Cefepim, Imipenem/Cilastatin oder Meropenem zur Verfügung. Bei den Fluorchinolonen wird von der American Thoracic Society Ciprofloxacin empfohlen (Abb.3). kurzgefasst: besondere Bedeutung und allgemeine Akzeptanz für die Therapie nosokomialer Pneumonien hat die Klassifizierung der American Thoracic Society (ATS) aus dem Jahr 1995. Die beatmungsassoziierte Pneumonie gehört definitionsgemäß zu den schweren Verlaufsformen.

Diese bisher vorliegenden Therapieempfehlungen der ATS haben das Problem der Differentialtherapie dargestellt und wichtige Therapieoptionen publiziert. In der täglichen Praxis erweist es sich jedoch als schwierig, diese auch umzusetzen. Aus diesem Grunde haben die Paul-Ehrlich-Gesellschaft (PEG) und die Deutsche Gesellschaft für Pneumologie (DGP) neue Therapieempfehlungen erarbeitet (4). Ziel dieser Empfehlungen ist es, einen auch für den nicht speziell infektiologisch ausgebildeten Kliniker verständlichen Leitfaden zu definieren. Es wurden 3 Therapieoptionen festgelegt, die aufgrund eines Punktesystems zum Einsatz kommen (Tab. 2).

Faktoren, die einer unterschiedlichen Gewichtung unterliegen (1-4 Punkte) sind: Alter, strukturelle Lungenerkrankung, antibiotische Vorbehandlung, später Manifestationszeitpunkt sowie der Schweregrad der Pneumonie. Beim Vorliegen mehrerer Einflussfaktoren werden die Punktwerte der Einzelfaktoren addiert. Aus der errechneten Punktzahl erfolgt eine Zuordnung der Patienten in drei unterschiedliche Therapieoptionen, wobei aufgrund der Punktwertung für die beatmungsassoziierte Pneumonie nur die Therapieoptionen II und III zur Anwendung kommen.

- In der Therapieoption II (3-5 Punkte) stehen Acylaminopenicilline in Kombination mit einem Betalaktamase-Inhibitor (Aufgrund der aktuellen Datenlage sollte in der Indikation respiratorassoziierte Pneumonie die fixe Kombination von Piperacillin mit Tazobactam bevorzugt zur Anwendung kommen, da nur diese in der Indikation nosokomiale Pneumonie durch eine Vielzahl von klinischen Studien gut dokumentiert ist), Cephalosporine der Gruppe 3b und Fluorchinolone der Gruppe 2 und 3 zur Verfügung. 


\section{Nosokomiale Pneumonien (Gruppe 3)*}

- schwere Erkrankung (inklusive beatmungsassoziierte Pneumonie), keine Risikofaktoren, „late onset“

- schwere Erkrankung (inklusive beatmungsassoziierte Pneumonie), mit Risikofaktoren, Beginn zu jeder Zeit

\begin{tabular}{|l|l|}
\hline \multicolumn{1}{|c|}{ zusätzliche Erreger } & \multicolumn{1}{c|}{ Antibiotikatherapie } \\
\cline { 1 - 2 } $\begin{array}{l}\text { Pseudomonas } \\
\text { aeruginosa }\end{array}$ & $\begin{array}{l}\text { Acylaminopenicillin/BLI+Ciprofloxacin } \\
\text { Cephalosporin Gruppe IIIb+Ciprofloxacin } \\
\text { Carbapenem + Ciprofloxacin }\end{array}$ \\
Acinetobacter spp. & $\begin{array}{l}\text { Ocylaminopenicillin/BLI+Aminoglykosid } \\
\text { Cephalosporin Gruppe IIIb+Aminoglykosid } \\
\text { Carbapenem + Aminoglykosid }\end{array}$ \\
falls MRSA $\longrightarrow$ & $\begin{array}{l}\text { eine der oben genannten ‘Kombinationen } \\
+ \text { Glykopeptid }\end{array}$ \\
\end{tabular}

Abb.3 Erreger nosokomialer Pneumonien und Therapie bei Patienten, die der Gruppe 3 zugeordnet werden (in Anlehnung an das Consensus Statement der American Thoracic Society (3)). Patienten mit beatmungsassoziierter Pneumonie werden definitionsgemäß den schweren Verlaufsformen zugeordnet (BLI: Betalaktamase-Inhibitor).

Tab.2 Kalkulierte Antibiotikatherapie der nosokomialen Pneumonie unter Berücksichtigung von Risikofaktoren.

\begin{tabular}{|c|c|c|}
\hline \multicolumn{3}{|l|}{ Therapie } \\
\hline I (bis 2 Punkte) & II (3-5 Punkte) & III (ab 6 Punkte) \\
\hline $\begin{array}{l}\text { Aminopenicillin/BLI } \\
\text { Cephalosporin 2/3a } \\
\text { Fluorchinolon 3/4 }\end{array}$ & $\begin{array}{l}\text { Acylamino- } \\
\text { penicillin/BLI } \\
\text { Cephalosporin 3b } \\
\text { Fluorchinolon 2/3 }\end{array}$ & $\begin{array}{ll}\text { Cephalosporin 3b } & \begin{array}{l}\text { Fluor- } \\
\text { chinolon 2/3 } \\
\text { Acylamino- } \\
\text { penicillin/BLI }\end{array} \text { oder Amino- } \\
\text { Carbapenem } & \text { glykosid }\end{array}$ \\
\hline
\end{tabular}

BLI: Betalaktamase-Inhibitor, DIC: Disseminierte intravasale Gerinnung, ANV: Akutes Nierenversagen, ALV Akutes Leberversagen

Risikofaktoren

Alter>65 Jahre

Strukturelle Lungenerkrankung

Antiinfektive Lungenerkrankung

„Late onset“ (Erkrankung ab dem 5. Tag Krankenhausaufent- 3

halt)

Schwere Respiratorische Insuffizienz mit oder ohne Beatmung 3

(maschinell oder nicht-invasiv)

Schwere Pneumonie, extrapulmonales Organversagen (Schock, 4

$D I C, A N V, A L V)$

- In der Therapieoption III (6 Punkte und mehr) ist grundsätzlich eine Kombinationstherapie erforderlich. Hier werden Cephalosporine der Gruppe 3b, Acylaminopenicilline in Kombination mit einem Betalaktamase-Inhibitor (s. o.) oder Carbapeneme vorzugsweise mit einem Fluorchinolon der Gruppe 2/3 oder mit einem Aminoglykosid kombiniert. kurzgefasst: Die neuen von der Paul-Ehrlich-Gesellschaft (PEG) und der Deutschen Gesellschaft für Pneumologie (DGP) entwickelten Therapieempfehlungen erlauben es auch einem nicht speziell infektiologisch ausgebildeten Kliniker, nosokomiale Pneumonien patientengerecht zu klassifizieren und zu behandeln. Hierbei handelt es sich um eine kalkulierte Therapie vor bzw. ohne Erregernachweis.

Diese Therapieempfehlungen beziehen sich ausschließlich auf die kalkulierte Antibiotikatherapie vor oder ohne Erregernachweis. Bei Nachweis von Pseudomonas spp. oder Acinetobacter spp. sollte abweichend von diesem Schema immer eine geeignete Kombinationstherapie durchgeführt werden. Traditionell sind Aminoglykoside die bevorzugten Kombinationspartner für Betalaktamantibiotika. Die neue Option, Fluorchinolone als bevorzugte Kombinationspartner für Betalaktamantibiotika einzusetzen, ist durch pharmakokinetische Vorteile, eine geringere Toxizität und die fehlende Notwendigkeit von regelmäßigen Spiegelbestimmungen trotz höherer direkter Therapiekosten begründet. In den Therapieoptionen II und III müssen alle Antibiotika parenteral und in hoher Dosierung appliziert werden, da weder die Empfehlung einer Sequenzialtherapie noch einer Dosisreduktion in dieser Indikation durch Studien belegt sind $(5,6)$.

\section{Literatur}

1 Bodmann KF. Therapie nosokomialer Infektionen. Uni-Med Verlag, Bremen - London - Boston, In: Bodmann KF. Therapiemanagement nosokomialer Infektionen. 2001

2 Fagon JY, Chastre J, Vuagnat A, Trouillet JL, Novara A, Gibert C. Nosocomial pneumonia and mortality among patients in intensive care units. JAMA 1996; 275: 866-869

3 American Thoracic Society. Hospital acquired Pneumonia in Adults. Diagnosis, Assessment of Severity, Initial Antimicrobial Therapy, and Preventative Strategies. Am J Resp Crit Care Med 1995; 153: 17111725

4 Bodmann K-F, Lorenz J, Bauer TT, Ewig S, Trautmann M, Vogel F. Nosokomiale Pneumonie. Prävention Diagnostik und Therapie. Ein Konsensuspapier der PEG und der DGP. Chemotherapie Journal 2002; in press

5 Bodmann KF, Vogel F. Antimikrobielle Therapie der Sepsis. Chemotherapie Journal 2001; 10: 43-55

6 Ewig S, Dalhoff K, Lorenz J, Schaberg T, Welte T, Wilkens H. Nosokomiale Pneumonie: Empfehlungen zur Therapie und Prophylaxe. Pneumologie 2000; 54: 525-538 